\title{
BMJ Open Degree of personalisation in tailored activities and its effect on behavioural and psychological symptoms and quality of life among people with dementia: a systematic review and meta- analysis
}

\author{
Shiyu Lu (D) , ${ }^{1}$ Anna Y Zhang, ${ }^{2}$ Tianyin Liu, ${ }^{2}$ Jacky C P Choy, ${ }^{2}$ Maggie S L Ma, ${ }^{2}$ \\ Gloria Wong (D) , ${ }^{3}$ Terry Lum (D) ${ }^{2}$
}

To cite: Lu S, Zhang AY, Liu T, et al. Degree of personalisation in tailored activities and its effect on behavioural and psychological symptoms and quality of life among people with dementia: a systematic review and meta-analysis. BMJ Open 2021;11:e048917. doi:10.1136/ bmjopen-2021-048917

- Prepublication history and additional supplemental material for this paper are available online. To view these files, please visit the journal online (http://dx.doi.org/10.1136/ bmjopen-2021-048917).

Received 12 January 2021 Accepted 21 October 2021

D Check for updates

(C) Author(s) (or their employer(s)) 2021. Re-use permitted under CC BY-NC. No commercial re-use. See rights and permissions. Published by BMJ.

For numbered affiliations see end of article.

Correspondence to

Professor Terry Lum;

tlum@hku.hk

\section{ABSTRACT}

Objectives To understand and assess the degree of personalisation of tailored activities for people with dementia (PWD); and to estimate the magnitude of the effects of levels of personalisation on reducing behavioural and psychological symptoms of dementia (BPSD), improving quality of life $(\mathrm{Q} o \mathrm{~L})$ and level of engagement. Design Systematic review with meta-analysis.

Data sources ProQuest, PubMed, Ovid, Cochrane Library, Web of Science and CINAHL were searched from the start of indexing to May 2020.

Eligibility criteria We included randomised controlled trials and quasi-experimental studies assessing the effects of tailored activities for people aged 60 years or older with dementia or cognitive impairment on the outcomes of BPSD, QoL, depression and level of engagement with control groups.

Data extraction and synthesis Two researchers screened studies, extracted data and assessed risks of bias. A rating scheme to assess the degree of personalisation of tailored activities was developed to classify tailored activities into high/medium/low groups. Effect sizes were expressed using standardised mean differences at 95\% Confidence Interval (Cl). Subgroup analyses were conducted to assess whether the degree of personalisation of tailored activities affected outcomes of interest.

Results Thirty-five studies covering 2390 participants from 16 countries/regions were identified. Studies with a high-level of personalisation interventions $(n=8)$ had a significant and moderate effect on reducing BPSD (standardised mean differences, $\mathrm{SMD}=-0.52, \mathrm{p}<0.05$ ), followed by medium ( $n=6$; SMD $=-0.38, p=0.071)$ and low-level personalisation interventions $(n=6 ; S M D=-0.15$, $p=0.076$ ). Tailored activities with a high-level of personalisation had a moderate effect size on improving QoL $(n=5 ; S M D=0.52, p<0.05)$, followed by a medium level $(n=3$; SMD $=0.41, p<0.05)$ of personalisation. Conclusions To develop high-level tailored activities to reduce BPSD and improve QoL among PWD, we recommend applying comprehensive assessments to
Strengths and limitations of this study

- The major contribution of this systematic review and meta-analyses is developing a rating scheme to assess the level of personalisation for interventions.

- To assess whether the degree of personalisation of the tailored activities affects reduction of behavioural and psychological symptoms of dementia and improves quality of life among people with dementia or cognitive impairment.

- Exclusion of papers not published in English may mean that important additional findings are missed.

identify and address two or more PWD characteristics in designed tailored activities and allow modification of interventions to respond to changing PWD needs/ circumstances.

PROSPERO registration number CRD42020168556.

\section{INTRODUCTION}

Dementia is particularly common among older adults, affecting $5 \%-8 \%$ of people aged 60 and over at any given time worldwide. ${ }^{1}$ Behavioural and psychological symptoms of dementia (BPSD) are common among people living with dementia (PWD), such as agitation, depression and resistance to care, ${ }^{2}$ which occur throughout the disease process, associated with decreased quality of life (QoL). ${ }^{3}$

Non-pharmacological interventions are recommended as first-line treatments over pharmacological approaches to treat BPSD and have less adverse effects. ${ }^{4}$ Tailored activities for PWD are promising nonpharmacological approaches that reduce BPSD and increase QoL. Two recently published National Institute for Health and 
Care Excellence (NICE) guidelines recommend that healthcare professionals offer activities to promote QoL that are tailored to personal preferences and consider using a structured tool to assess their likes, dislikes, routines and personal history. ${ }^{56}$

To our knowledge, six systematic reviews and metaanalyses (summarised in online supplemental table 1) have synthesised the effects of tailored activities on reducing BPSD and enhancing QoL among PWD, based on tailored strategies, activity types, personal characteristics, and frequency and duration of delivery. ${ }^{7-12}$ The first of these, incorporating studies published between 2000 and 2011, focused on the effectiveness of various tailored strategies to foster activity engagement and reduce BPSD in PWD. ${ }^{7}$ Changes to tools and materials used in activities were most common but yielded mixed outcomes of BSPD reduction; modifications to space and social demands were rarely tested but yielded consistently positive outcomes. ${ }^{7}$ In addition, a systematic review of studies published between 2000 and 2012 found that personalised pleasant activities yielded strong evidence for treating BPSD but limited evidence for physical and music activities. ${ }^{8}$ Another meta-analysis found that individualised recreational activities were effective for reducing BPSD. ${ }^{9}$ Recently, Möhler and colleagues conducted three meta-analyses regarding the effects of tailored activities among PWD living in care facilities, communities and home settings, respectively, and found that, compared with usual care, tailored activities slightly reduced BPSD. ${ }^{10-12}$ However, no differences in other desired outcomes between intervention and control groups among different specific types of activity or duration of delivery were evident. Although different activity components (eg, activity types, PWD characteristics, frequency and duration of delivery) were discussed ${ }^{7-12}$ no review further investigated the degree of personalisation among the tailored activities and synthesised its associations with the desired outcomes.

Understanding the degree of personalisation of tailored activities is important. We define the degree of personalisation of tailored activities as the extent to which non-pharmacological interventions are tailored, individualised or personalised for PWD. The conceptualisation of the degree of personalisation echoes the rationales and principles of effective interventions working on BPSD, level of engagement and QoL, embedded in occupational therapy, ${ }^{13}$ engagement in meaningful activities $^{14}$ and person-centred care. ${ }^{15}$ Occupational therapy emphasises the fit between PWD capabilities and the occupation (eg, activities or roles) through task simplification and removing barriers in the physical and social environment. ${ }^{13}$ Environmental docility theory suggests that both underloading and overloading of external stimulations (e.g., cognitive activities and social interactions) may lead to PWD disengagement or excessive disability. ${ }^{16}$ Thus, maintaining PWD engagement in meaningful activities through tailored activities based on their physical strength, mental state and psychosocial needs is essential. ${ }^{14}$ The person-centred care approach stresses service providers' and caregivers' autonomy to determine specific ways of delivering care to maintain participants' engagement during the intervention. ${ }^{15}$ These theories imply that the degree of personalisation can significantly influence the effectiveness of tailored activities for PWD. Thus, the degree of personalisation could depend on the assessment of PWD characteristics and their environment, the design of tailored activities based on PWD characteristics, and interventionists' autonomy to address PWD spontaneous needs.

Conceptualising and quantifying the levels of personalisation of existing tailored activities can advance our knowledge on developing a high level of personalisation of tailored activities for PWD, deciding on the appropriate 'dose' of tailoring, and translating this cumulative evidence into clinical practice. However, existing literature provides little knowledge about assessing the degree of personalisation among tailored activities and their effectiveness on targeted outcomes.

\section{Objectives}

This systematic review and meta-analysis aimed to: (1) assess the degree of personalisation of existing tailored activities for PWD; (2) estimate the magnitude of the effects of existing tailored activities on reducing BPSD, improving QoL and the level of engagement among PWD and (3) assess whether the degree of personalisation of tailored activities affects the outcomes of interest.

\section{METHODS AND ANALYSIS}

We conducted the review in accordance with the Preferred Reporting Items for Systematic Reviews and Meta-Analyses (PRISMA) procedure. ${ }^{17}$ Eligibility criteria required studies to: (1) include participants with dementia or cognitive impairment and aged 60 years or older; (2) include activities tailored to at least one of the participants' characteristics (eg, needs, physical or/ and mental ability, present or previous preferences for particular activities or interests, habits and physical living environments like housing conditions and caregiver management style); (3) report BPSD (measured by multidomain scales, such as the Neuropsychiatric Inventory (NPI), and scales specific to agitation and depression/ anxiety, such as the Cohen-Mansfield Agitation Inventory and the Cornell Scale for Depression in Dementia), ${ }^{18-20}$ QoL and level of engagement as outcomes; (4) include randomised controlled trials or quasi-experimental study design and (5) apply a control group (CG) (eg, usual care, wait-list, attention control, etc). The review included studies published in English from the start of indexing to May 2020.

We searched ProQuest (e.g., APA PsycInfo), PubMed, Ovid (e.g., Embase), Cochrane Library, Web of Science and CINAHL, using the search terms: (1) "cognitive impairment" OR "cognitive disorder" OR "dement*" OR "Alzheimer"; (2) "tailor*" OR "engag*" OR "individual*" OR "personal*"; and (3) "activit*" OR "program*" OR 
"therap*" OR "intervention*" OR "treatment*". The full search strategy is shown in online supplemental table 2.

SL and AYZ independently completed the title/abstract review and full-text review. We conducted title/abstract screening using Rayyan (https:/ / www.rayyan.ai/) and fulltext review using Endnote. The two researchers discussed disagreements in the title/abstract screening and fulltext review to reach consensus. Data were extracted and checked by SL and MSLM. Where there were disagreements, data were rechecked for relevance and accuracy. Where available, raw data (eg, clinical interventions, strategies, outcomes and results) were extracted and entered into a spreadsheet. ${ }^{21}$ For each intervention, we additionally extracted the following information: PWD (including older people with cognitive impairment) characteristics taken into account, intervention delivery, and information about the tailoring process (the data extraction form is shown in online supplemental appendix 1).

\section{Patient and public involvement}

No patient involved.

\section{Developing the tailoring and classification scheme}

The authors formed an expert panel to develop a scheme for the level of personalisation interventions based on the included studies, comprising AYZ (a licensed social worker in Hong Kong with 2 years clinical experience of dementia care and 5 years research experience focusing on the mechanisms of non-pharmacological interventions for PWD), TLiu, JCPC and SL (each of whom had over 10-year experience in psychology and elderly care).

Based on the theories and approaches mentioned above, we hypothesised that tailoring is embedded in the whole process at three inter-related phases: assessment, design and implementation, and the degree of personalisation is determined by these three dimensions: (1) how to assess PWD characteristics before designing the intervention; (2) the extent to which interventions are tailored according to PWD characteristics and (3) the level of the interventionists' autonomy to address PWD needs, as suggested by occupational therapy, engagement in meaningful activities, and the person-centred care approach (online supplemental figure 1). ${ }^{13-15}$ To this end, we developed three corresponding criteria to rate levels of personalisation (online supplemental table 3).

First, the level of assessment for tailoring refers to how comprehensive the PWD characteristics were considered and how systematically the assessment results were used for designing tailored activities. Operationally, we rated the level of assessment as 'unclear/incomprehensive', 'semi-structured' or 'structured'. 'Unclear/incomprehensive' indicated that preassessment was missing/not clearly described, only a single domain of PWD characteristics was assessed, or no description of how the assessment results were used to inform the tailored activities design. 'Semi-structured' referred to preassessments conducted by unstructured/semi-structured interviews, with some descriptions on how the assessment results were used for activities design, 'structured' preassessments employed structured interviews with clear and detailed descriptions on how the assessment results were systematically used for the activities design.

Second, individualisation in intervention design refers to how the intervention design accounted for individuals' uniqueness and variations of their needs. To avoid counting the number or arbitrarily weighting specific PWD characteristics, we distinguished the degree of individualisation based on whether the protocol tailored for one versus two or more PWD characteristics.

Third, the degree of person-centred care in implementation refers to how the interventionists were able to adjust the intervention based on their clinical knowledge and observation of participants' performance in the intervention to maintain participants' engagement and respond to participants' spontaneous needs during the implementation. Intervention with a standardised protocol of tailored activities regardless of spontaneous needs of PWD were rated as low flexibility for pursuing person-centred care, and interventions encouraging and allowing great flexibility for interventionists to adjust the tailored activities based on clinical knowledge and observation of participants' performance were rated as high flexibility.

Based on the dimensions mentioned above, we rated the level of personalisation of tailored activities as high, medium or low. A study was rated as high level only if it met all the following criteria: (1) structured assessments were used for systematically tailored activities plan; (2) interventions targeted two or more domains (eg, capabilities, preferences, interests, life experience and external environment) and (3) allowed the interventionists to exercise flexibility to adjust the intervention in accordance with PWD spontaneous needs. A study was rated as medium if: (1) unstructured/semi-structured assessments on participants' characteristics were performed; (2) interventions targeted two or more domains and (3) some flexibility and modifications were allowed for adjusting the intervention in response to PWD needs. A study was rated as low if: (1) assessment was unclear/ incomprehensive, or there was no clear description on how assessment results informed tailoring; (2) interventions targeted only one domain of participants' characteristics and (3) low/marginal flexibility to pursue person-centred care for interventionists was allowed. AYZ and SL independently rated the level of personalisation for the included tailored activities. The inter-rater reliability was $88.8 \%$ in the initial stage of rating. Conflicting ratings were resolved through discussion.

\section{Data synthesis and analysis}

Given that outcomes in our review were continuous, effect sizes were expressed using standardised mean differences (SMD) at 95\% CI, ${ }^{20}$ interpreted as Cohen's d. ${ }^{22}$ Specifically, the values of $0.2,0.5$ and 0.8 reflected small, moderate and large effect sizes, respectively. ${ }^{22}$ Due to differences in settings and methods, we used the 
random-effects model to pool the results. Heterogeneity was determined by $\chi^{2}$ and $\mathrm{I}^{2}$ statistics. ${ }^{23}{ }^{24}$ We classified subgroup analyses of the effectiveness of tailored activities according to the levels of personalisation of the interventions. All meta-analyses were conducted using Comprehensive Meta-Analysis Software. Where raw data are not provided, summary results are given in the text but not the forest plots. The meta-analyses included results from randomised controlled studies (RCTs) only because the findings from quasi-experimental studies were not comparable to those from RCTs. Sensitivity analyses were conducted to check the robustness of the findings.

\section{Quality appraisal}

SL and MSLM independently assessed the risk of bias for the studies using a revised Cochrane risk of bias tool for randomised trials, ${ }^{23} 25$ including: (1) bias arising from the randomisation process; (2) deviations from intended interventions; (3) bias due to missing outcome data; (4) bias in measurement of the outcome and (5) bias in selection of the reported results. Risk of Bias in Nonrandomised Studies of Interventions was used to categorise the risk of bias as 'low,' 'high' or 'some concerns' for non-RCT studies. ${ }^{22}$ Conflicting results were resolved through discussions.

\section{RESULTS}

\section{Summary of search results}

The search and study selection process is summarised in the PRISMA flow diagram (online supplemental figure 2). In the identification phase, 14238 abstracts were identified and imported into Endnote; 7471 duplicate articles were removed. In the screening phase, the titles and abstracts of 6767 articles were screened, and 6476 irrelevant articles were excluded. In the eligibility phase, fulltext screening was conducted for 291 articles according to the inclusion and exclusion criteria, and 35 studies were finally included in this review.

Included studies were conducted in 16 countries/ regions: Australia, Brazil, Mainland China, Denmark, France, Germany, Hong Kong, Italy, Japan, Korea, the Netherlands, Norway, Switzerland, Taiwan, the UK and the USA, published between 2000 and 2020. The average age of participants ranged from 62.1 to 89.2 years. Twenty-nine studies included participants with dementia only, and the remaining studies included participants with mild to moderate levels of cognitive impairment. The total size of the intervention groups (IGs) was 1248 (range $=6-158$ ), and the total size of the CGs was 1142 (range $=5-107)$. Fourteen studies $(40 \%)$ had no more than 20 participants for each arm. Thirty studies were RCTs. Five applied a quasi-experimental study design. Twenty-two applied usual care as the comparison, and the remaining applied placebo control, active control or wait-list control. Twenty-four studies were conducted in care facilities (such as a nursing home, geriatric health service facility or hospital), and the remaining studies were conducted in community settings or home-based settings (online supplemental table 4).

\section{Description of the interventions}

The components of activities can be categorised into four groups: physical $(n=3),{ }^{26-28}$ cognitive $(n=2),{ }^{29}{ }^{30}$ music $(\mathrm{n}=7)^{31-37}$ and multiple activities $(\mathrm{n}=23) .{ }^{16}{ }^{38-59}$ Twentythree studies reported their interventions as individual mode, five reported group-based mode and six reported mixed modes, while the remaining studies did not provide details. Intervention was provided by specialists (eg, occupational therapists, clinicians, psychologists, physical therapists and speech therapists), researchers and trained nursing home caregivers and staff. A detailed description of interventions is shown in online supplemental table 5 .

\section{Level of personalisation}

Based on the three-dimension rating scheme for the personalisation of tailored activities, we identified 12 studies as high level, ${ }^{16} 2635394046495153-5611$ as medium $^{3436} 38424445475257-59$ and 11 as low. ${ }^{27-33} 37434850$ One was rated as mixed because it had three-arm IGs with one medium and two low levels of tailoring activities for comparison. ${ }^{41}$ Table 1 shows the level of personalisation among the interventions reported in the reviewed studies.

\section{Level of assessment for tailoring}

Sixteen studies assessed the full picture of PWD characteristics using structural assessments. ${ }^{16} 263538-4246495153-5659$ For instance, five studies followed the protocol of the Tailored Activity Programme (TAP) incorporating the Progressive Lowered Stress Threshold Model. ${ }^{60}$ This posits that with disease progression, dementia patients become increasingly vulnerable to their environment and experience lower thresholds for tolerating stimuli that can result in behavioural disturbances. TAP applied systematic approaches to discern PWD and their caregivers' daily routines, identify previous and current activity interests and collect information about dyadic communication and home environmental features to design activities for participants.

\section{Degree of individualisation in design}

Activities tailored according to PWD characteristics included cognitive or/and physical capacities $(\mathrm{n}=22),{ }^{26-28} 3038 \quad 3941-4749 \quad 51 \quad 53-59$ personal experience and history $(\mathrm{n}=2),{ }^{3448}$ role identity $(\mathrm{n}=3), 395155$ preferences and interests $(\mathrm{n}=20)^{26} 29$ 31-33 35-37 40-42 4647 50 53-57 59 habits $(\mathrm{n}=2),{ }^{51}{ }^{54}$ cultural backgrounds $(\mathrm{n}=1)^{47}$ and living environment $(n=5) .{ }^{49} 51535456$ Five studies also considered caregivers' characteristics. ${ }^{46} 51535456$ Twelve studies tailored the intervention for a single aspect of PWD characteristics only, while the remainder tailored the activities for at least two. One study used a four-arm study design (three IGs plus one CG), with one tailoring both for PWD capacity and interests, the second only tailoring for the capacities yet opposite to PWD preference, and the third only tailoring for the interests yet challenging to PWD' capacity in the three IGs. ${ }^{41}$ 


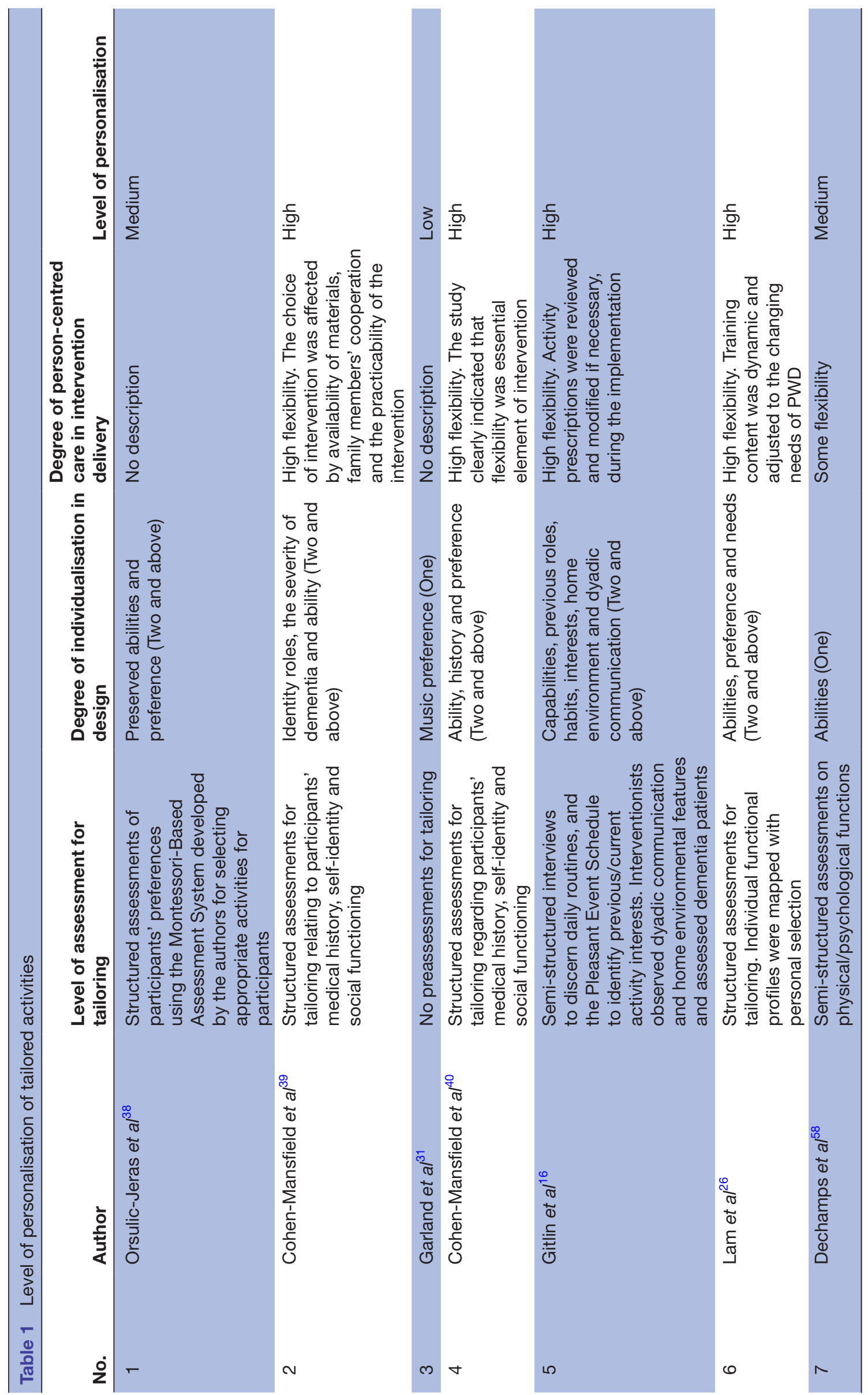




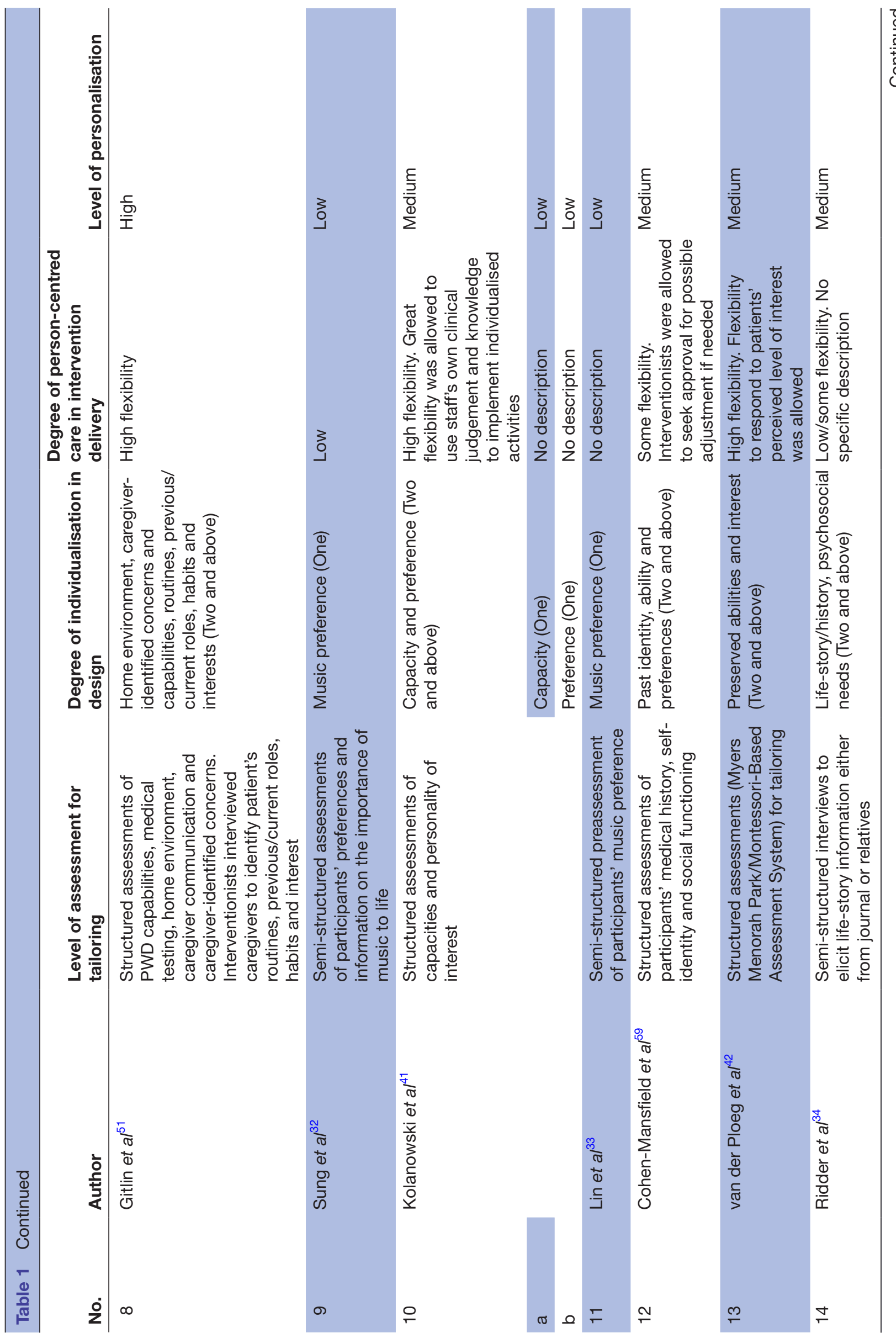




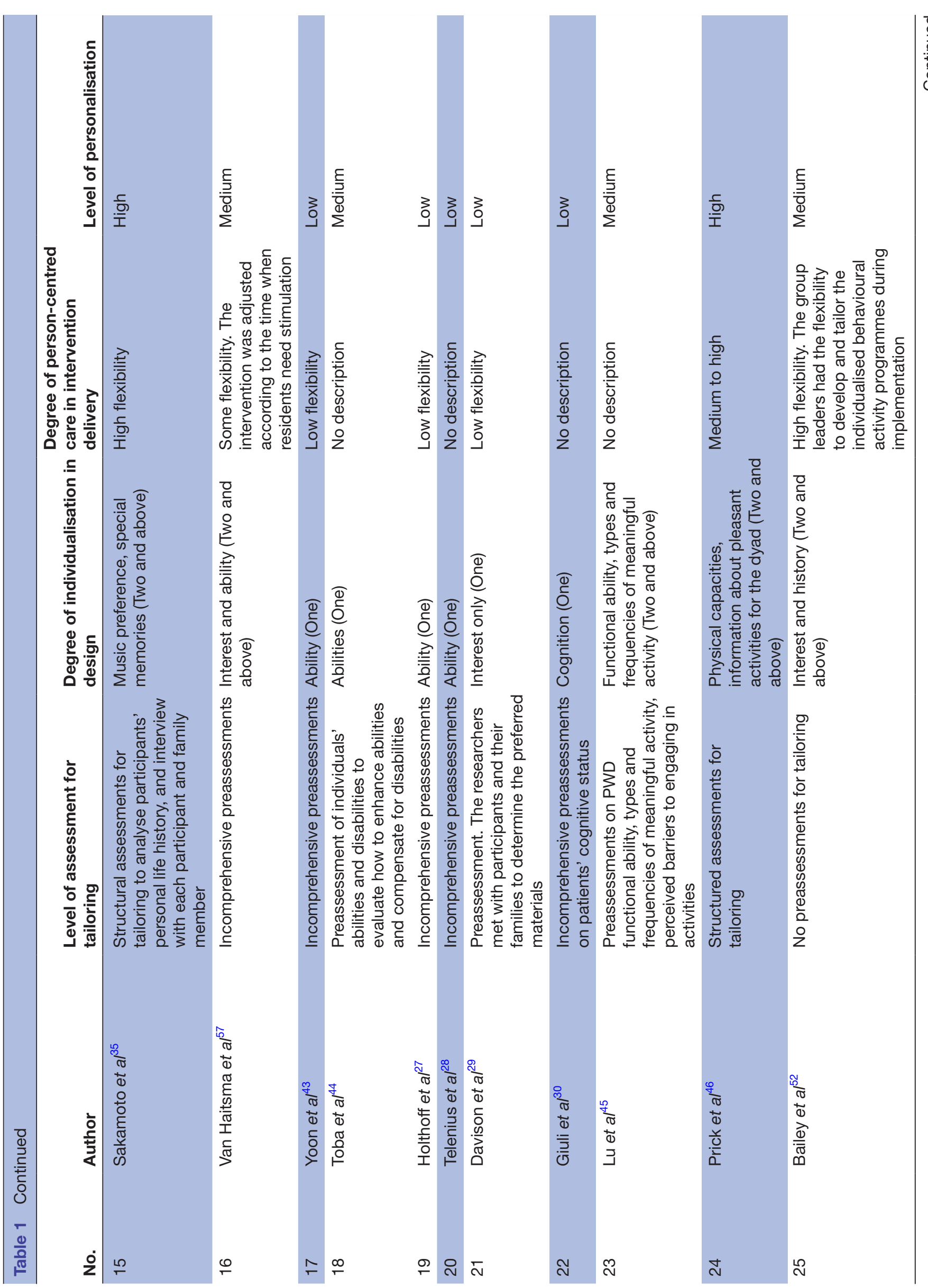



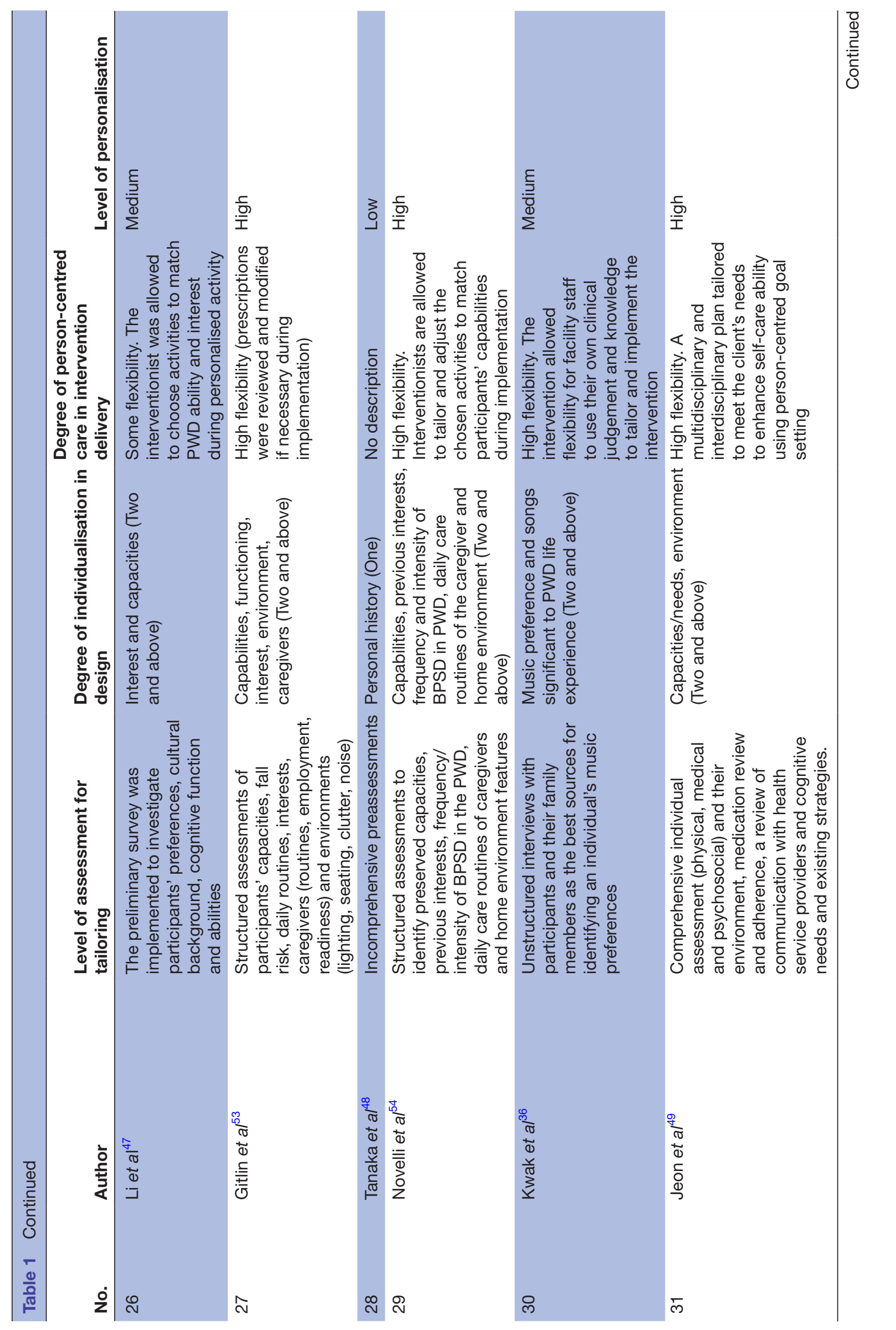

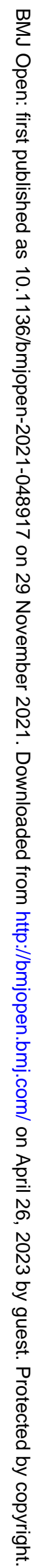




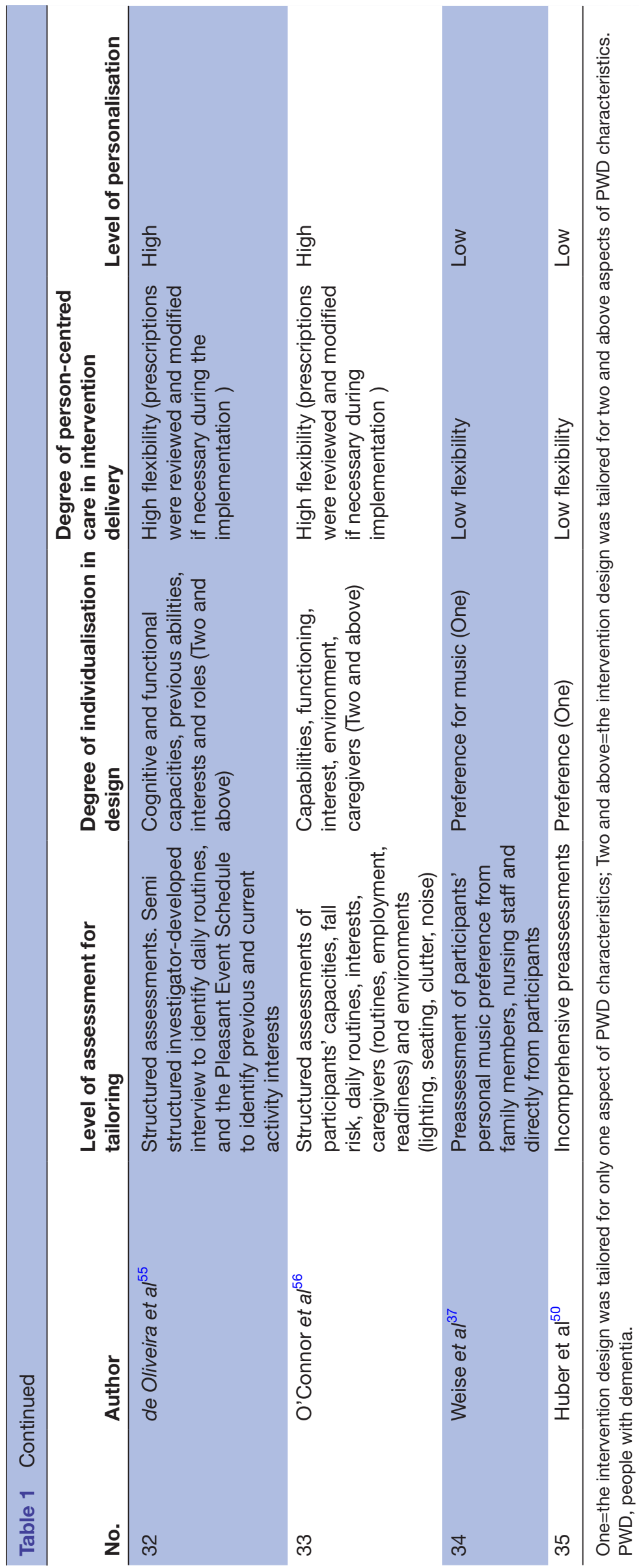




\section{Degree of person-centred care in delivery}

Twenty-six studies indicated the level of flexibility for modification of activities during the intervention. Sixteen studies explicitly permitted the interventionists to review and modify the intervention according to participants' spontaneous needs and circumstances, $1626353639-42464951-56$ thus were rated as offering a high degree of personcentred care. Five allowed some flexibility for adjusting interventions during implementation, ${ }^{34} 4757-59$ thus were rated as offering some flexibility. Five studies enabled relatively limited adjustment of intervention to take account of changed PWD needs or circumstances. ${ }^{27} 32343750$ The remaining studies provided insufficient information to judge the extent of flexibility allowed during the intervention.

\section{Quality appraisal}

The risk bias of 10 RCT studies was judged as low, while that of 12 was rated as high, and the remainder was judged as giving some concern (online supplemental figure 3). Nineteen RCT studies reported the method of random sequence generation (eg, computer-generated programmes, random list generator, random allocation by an external researcher and block randomisation). ${ }^{1628-3033343741-4346495153-565859}$ Eleven were rated as high risk of deviation from intended intervention as they were judged as high risk of blinding participants, personnel and appropriate analysis used to estimate the effect of assignment to intervention. ${ }^{27-30} 33364042434852$ Five quasi-experimental studies were excluded from the meta-analysis since none were rated at low risk of bias and thus comparable to RCTs (online supplemental figure 4).

\section{Meta-analysis: the effects of tailored interventions}

Twenty-six studies reported the outcomes of BPSD measured by multi-dimension or specific scales of agitation (figure 1). ${ }^{16} \quad 27-2931 \quad 33-37 \quad 39-42 \quad 44 \quad 46 \quad 47 \quad 50 \quad 51$ 53-59 The measurements used for BPSD included the NPI, the Agitation Behavior Mapping Instrument, the CohenMansfield Agitation Inventory, the Agitated Behaviors in Dementia Scale, the Behavioral Pathology in Alzheimer's Disease rating scale and the short version of the Dementia Behavior Disturbance Scale. ${ }^{182061-64}$ A higher score indicates more BPSD. According to our meta-analysis, 18 RCTs with 20 tailored activities had an overall small effect on BPSD at postintervention $\left(\mathrm{SMD}_{\text {pooled }}=-0.38 ; 95 \% \mathrm{CI}\right.$ -0.54 to $-0.22, \mathrm{p}<0.001)$, although significant heterogeneity was found $\left(\mathrm{I}^{2}=64.17 \%, \mathrm{p}<0.001\right)$. Eight studies were excluded from the meta-analysis either because of their quasi-experimental design or for not reporting the raw

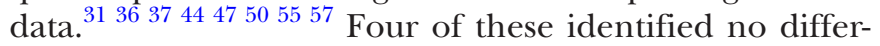
ences in reducing BPSD between IG and CG. ${ }^{31} 363750$

Nine studies reported the outcome of QoL (figure 2). ${ }^{163448495152545658}$ The measurements used for QoL included Quality of Life-Alzheimer's Disease, the 3-Level version of the EuroQol five dimensions, the EuroQol 5-D, and the Health-related Quality of Life Questionnaire for the Elderly with Dementia. ${ }^{65-67} \mathrm{~A}$ higher score indicates higher QoL. Tailored interventions had an overall small effect on QoL at postintervention $\left(\mathrm{SMD}_{\text {pooled }}=0.45 ; 95 \%\right.$ CI 0.25 to $\left.0.64, \mathrm{p}<0.001\right)$, and no significant heterogeneity was found $\left(\mathrm{I}^{2}=11.56 \%, \mathrm{p}>0.05\right)$.

Sixteen studies reported the outcome of depression, ${ }^{16} 26$ 28-30 3239 43-46 48-50 5258 measured by the Cornell Scale for Depression in Dementia, the Geriatric Depression Scale, the Multidimensional Observation Scale for Elderly Subjects, the Geriatric Depression Scale, the NPI subscale for depression or Patient Health Questionnaire-9. ${ }^{181968-70}$ A higher score indicates more depression. Thirteen RCT studies with 14 tailored activities indicated those activities had a small overall effect on depression at postintervention $\left(\mathrm{SMD}_{\text {pooled }}=-0.29 ; 95 \% \mathrm{CI}-0.45\right.$ to -0.13 , $\mathrm{p}<0.001)$, and no significant heterogeneity was found

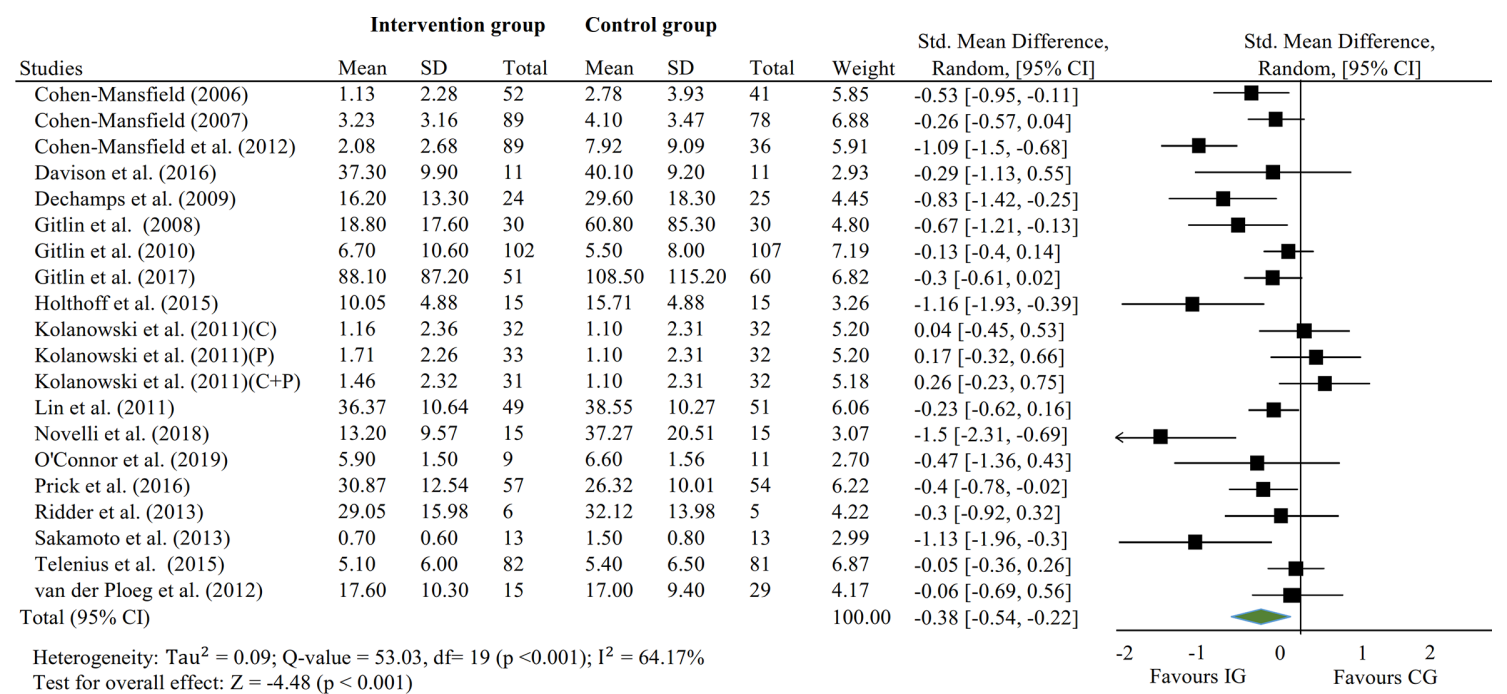

Figure 1 Effects of tailored interventions on challenging behaviour at postintervention $(\mathrm{N}=20)$. $\mathrm{C}=$ activities tailored for capacities of participants only, $\mathrm{P}=$ activities tailored for preference of participants only, $\mathrm{C}+\mathrm{P}=$ activities tailored for capacities and preference of participants. Fixed effect: $\mathrm{SMD}_{\text {pooled }}=-0.32,95 \% \mathrm{Cl}-0.42$ to $-0.22, \mathrm{p}<0.001$. CG, control group; IG, intervention group. 


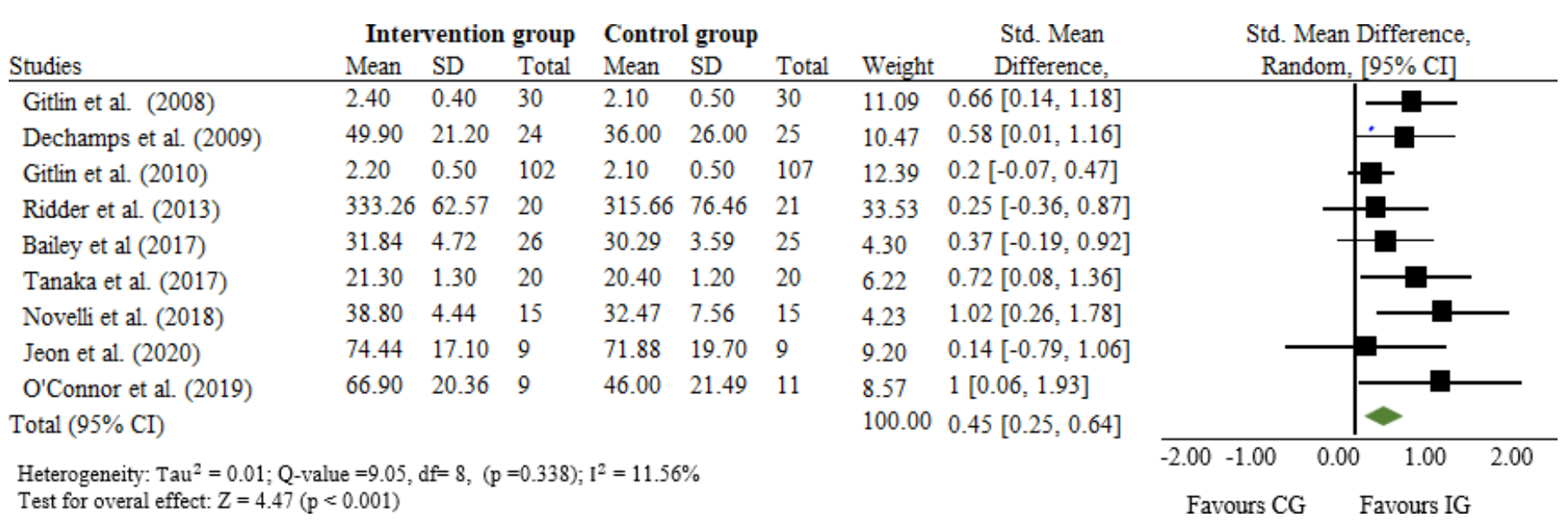

Figure 2 Effects of tailored interventions on quality of life at postintervention ( $N=9)$. Fixed effect: $\mathrm{SMD}_{\text {pooled }}=0.42,95 \% \mathrm{Cl} 0.24$ to $0.59, \mathrm{p}<0.001$. CG, control group; IG, intervention group.

(online supplemental figure 5). The remaining three studies were excluded from the meta-analysis because of their quasi-experimental design or lack of comparable data, ${ }^{324450}$ and only one study found no difference in reducing depression between IG and CG. ${ }^{44}$

Seven studies with nine interventions reported the outcome of engagement. ${ }^{16} 383941425152$ The measurements of engagement included one item on the ABMI, the Menorah Park Engagement Scale, direct observation or caregiver report. ${ }^{61} 71$ A higher score indicates a higher level of engagement. The meta-analysis indicated that tailored interventions of eight matched IGs and CGs in six studies had an overall large effect on the level of engagement at postintervention $\left(\mathrm{SMD}_{\text {pooled }}=0.86 ; 95 \%\right.$ CI 0.23 to $1.48, \mathrm{p}<0.001$ ) (online supplemental figure 6). Significant heterogeneity was found, primarily generated by the outlier study whose intervention specifically targeted participants' self-identity roles and which reported large effects on engagement (SMD=3.52; 95\% CI 2.87 to 4.17, $\mathrm{p}<0.001) .^{39}$ Removal of this study resulted in lower and non-significant heterogeneity with a significant small effect size $\left(\mathrm{SMD}_{\text {adjusted pooled }}=0.47 ; 95 \%\right.$ CI 0.23 to 0.60 , $\mathrm{p}<0.001)$. One study with a quasi-experimental design reported increased engagement postintervention. ${ }^{38}$

\section{Subgroup analysis}

Subgroup analysis was performed to test the difference of the effects of tailored activities with different levels of personalisation on outcomes (figures 3 and 4). Studies with a high level of personalisation tailored activities had a significant and the largest effect size regarding the reduction of $\mathrm{BPSD}\left(\mathrm{SMD}_{\text {pooled }}=-0.52,95 \% \mathrm{CI}-0.74\right.$ to $-0.29, \mathrm{p}<0.001)$ with non-significant heterogeneity, followed by medium $\left(\mathrm{SMD}_{\text {pooled }}=-0.38,95 \% \mathrm{CI}-0.79\right.$ to $0.03, \mathrm{p}=0.071)$ and low groups $\left(\mathrm{SMD}_{\text {pooled }}=-0.15,95 \% \mathrm{CI}\right.$ -0.44 to $0.14, \mathrm{p}=0.076$ ), although both the latter two groups had marginally significant effect sizes and significant heterogeneity. The high group had a moderate effect size on improvement in QoL $\left(\mathrm{SMD}_{\text {pooled }}=0.52\right.$, $95 \%$ CI 0.16 to $0.89, \mathrm{p}<0.01)$, followed by the medium group $\left(\mathrm{SMD}_{\text {pooled }}=0.41,95 \%\right.$ CI 0.07 to $\left.0.74, \mathrm{p}<0.05\right)$. Only one study with a low level of personalisation tailored activities reported the outcome of QoL with moderate effect size $(\mathrm{SMD}=0.72,95 \%$ CI 0.08 to $1.36, \mathrm{p}<0.05)$.

Subgroup analysis was performed to test the difference of the effects of the level of personalisation on depression and engagement (online supplemental figures 7 and 8). The medium group had a moderate effect size regarding reduction in depression $\left(\mathrm{SMD}_{\text {pooled }}=-0.64,95 \% \mathrm{CI}-1.14\right.$ to $-0.15, \mathrm{p}<0.05)$, followed by the high group $\left(\mathrm{SMD}_{\text {pooled }}=-0.33\right.$, $95 \%$ CI -0.54 to $-0.12, \mathrm{p}<0.01)$. The three studies with a medium level of personalisation of tailored activities all involved social or group interaction components that have beneficial effects on PWD mental health. Only one study rated high on tailoring had a large effect on improving engagement level postintervention $(\mathrm{SMD}=0.85$, $95 \%$ CI 0.32 to $1.38, \mathrm{p}<0.01)$. The medium group had a small effect size $\left(\mathrm{SMD}_{\text {pooled }}=0.44,95 \%\right.$ CI 0.07 to $\left.0.80, \mathrm{p}<0.05\right)$, followed by the low group $\left(\mathrm{SMD}_{\text {pooled }}=0.39,95 \% \mathrm{CI} 0.04\right.$ to $\left.0.74, \mathrm{p}<0.05\right)$.

\section{Sensitivity analysis}

We conducted a series of sensitivity analyses that excluded studies that combined participants with dementia and those with cognitive impairment. No substantial differences were found between the findings of studies focussing exclusively on PWD and studies that included participants with dementia and participants with cognitive impairment (online supplemental table 6). Sensitivity analyses were also conducted to examine whether the effect sizes of tailored activities on the outcomes of interest were associated with each study's sample size. The only significant association was found between sample size and effect size on QoL. We also tested whether a study's intervention mode (individual, group and mixed with mixed mode set as the reference group) would be associated with its findings. No significant associations were found between intervention mode and the outcomes.

\section{DISCUSSION}

Our systematic review aimed to assess the degree of personalisation of tailored activities and estimate the effect of levels of personalisation of tailored activities 


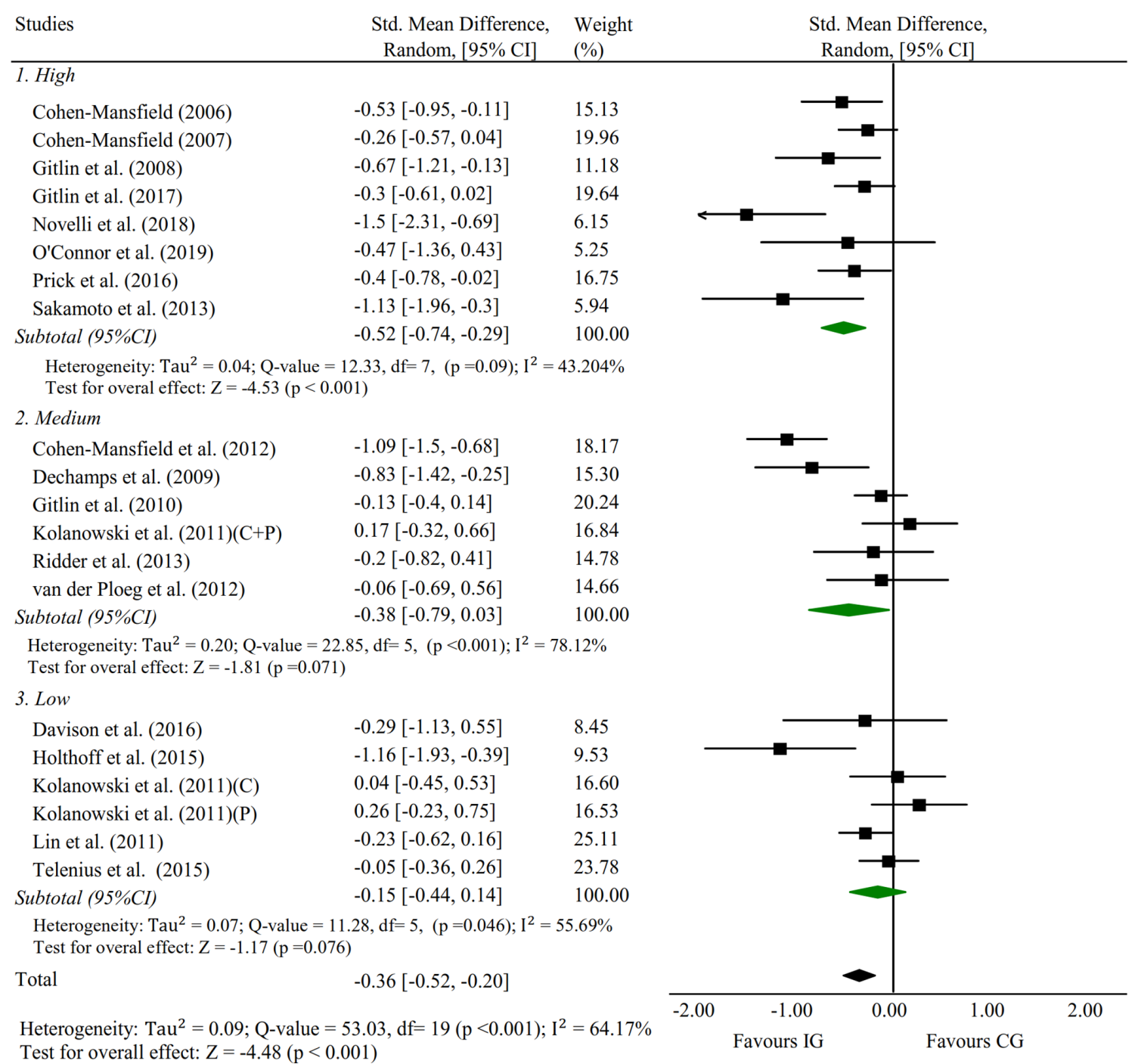

Figure 3 Subgroup analysis: effects of tailored interventions on challenging behaviour at postintervention by level of personalisation $(\mathrm{N}=20)$. $\mathrm{C}=$ activities tailored for capacities of participants only, $\mathrm{P}=$ activities tailored for preference of participants only, $\mathrm{C}+\mathrm{P}=$ activities tailored for capacities and preference of participants. (1) High group. Fixed effect: $\mathrm{SMD}_{\text {pooled }}=-0.46,95 \% \mathrm{Cl}$ -0.62 to $-0.30, p<0.001$; middle group. Fixed effect: $\mathrm{SMD}_{\text {pooled }}=-0.34,95 \% \mathrm{Cl}-0.51$ to $-0.16, \mathrm{p}<0.001$; low group. Fixed effect: $S M D_{\text {pooled }}=-0.11,95 \% \mathrm{Cl}-0.30$ to $0.08, p=0.254$. (2) Test for the difference across three subgroups: $Q$ value $=7.78, d f(Q)=2, p$ value=0.02. $\mathrm{CG}$, control group; IG, intervention group.

on reducing BPSD, improving QoL and other relevant outcomes among PWD. Thirty-five studies met our inclusion criteria, covering a total of 2390 participants from 16 countries/regions. The activities included in the interventions comprised physical, cognitive, music and multiple activities.

We employed meta-analysis to estimate the overall effects of tailored activities on the outcomes of BPSD, QoL, depression and engagement. Our findings on the effect sizes of tailored activities of the outcomes of interests differ from previous review studies. First, we found that tailored activities slightly reduced BPSD, consistent with previous meta-analyses targeting facilities, communities and PWD living in their own home. ${ }^{10-12}$ Second, we found that tailored activities had a small effect on improving QoL, compared with previous reviews that found inconclusive evidence regarding QoL: no effect in facilities, and a slight improvement in both communitybased and home-based settings. ${ }^{10-12}$ Third, our findings showed that tailored activities had small effects on depression, and large effects on engagement, contradicting previous reviews reporting little or no effect on these outcomes. ${ }^{11} 12$

Unlike previous review studies, we further developed the rating scheme of tailoring level based on three essential components: assessment for tailoring, individualisation in intervention design and person-centred care in implementation. Based on our rating scheme, the activities with optimal tailoring conditions possess the following characteristics. In the assessment stage, systematic interviews on individuals' characteristics were conducted. In the design stage, two or more domains of individuals' characteristics were targeted in the activity plan, including capabilities, preferences, interests, life 


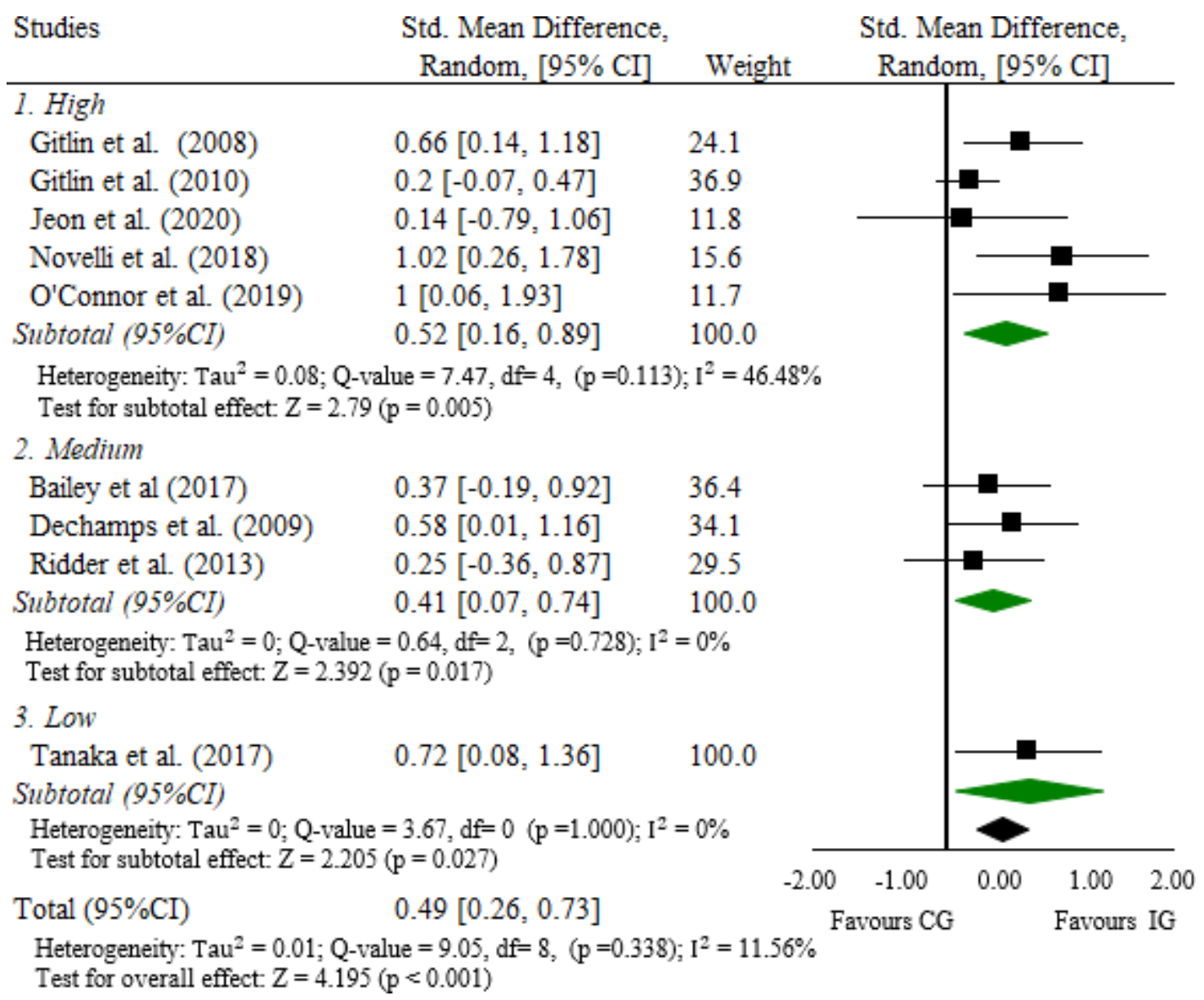

Figure 4 Subgroup analysis: effects of tailored interventions on quality of life at postintervention by level of personalisation $(\mathrm{N}=9)$. High group, fixed effect: $S M D_{\text {pooled }}=0.39,95 \% \mathrm{Cl} 0.17$ to $0.60, p<0.001$; middle group, fixed effect: $\mathrm{SMD}_{\text {pooled }}=0.41,95 \% \mathrm{Cl}$ 0.07 to $0.74, p=0.017$; low group, fixed effect: $S M D_{\text {poled }}=0.72,95 \% \mathrm{Cl} 0.08$ to $1.36, p=0.027$. (2) Test for the difference across three subgroups: $Q$ value $=0.94, \mathrm{df}(\mathrm{Q})=2, \mathrm{p}$ value $=0.626$. $C G$, control group; IG, intervention group.

experience and external environment. In the implementation stage, interventionists were allowed high flexibility and any modifications based on their professional judgement to accommodate the spontaneous needs of PWD during the intervention. Overall, we rated only 12 studies as high level of personalisation of tailored activities, 11 as medium, 11 as low and 1 study was rated as mixed because it had three-arm IGs with one medium and two low levels of tailoring activities for comparison.

Based on our rating scheme, we extended previous review studies to investigate how the degree of tailoring influenced intervention effectiveness on the outcomes of interest. Interventions with a high level of personalisation of tailored activities had a significant and moderate effect, followed by medium (small) and low groups (trivial); the latter two groups had significant heterogeneity and marginally significant effect sizes. Interventions rated as having a high level of personalisation had a moderate effect size on improving QoL, followed by the medium group. Only one study with a low level of personalisation of tailored activities reported the outcome of QoL with moderate effect size. These findings support our rating scheme as the overall goals of tailoring activities are to reduce BPSD and improve QoL. ${ }^{54}{ }^{56} \mathrm{~A}$ similar pattern was found in the level of engagement. However, because the degree of personalisation was rated high in one study only, this should be interpreted with caution.

This systematic review has several limitations. The generalisability of our results may be limited since we included English-language studies only. The included studies had risks of bias that may undermine the quality of evidence. Furthermore, noticeable heterogeneity was found among studies with outcomes of BPSD and engagement, which may affect the conclusions synthesised from these studies. Thus, these results must be interpreted with caution. In addition, the rating scheme for the level of personalisation was subjective regarding the level of assessments for tailoring and the degree of person-centred care in implementation.

This review has implications for clinical practice. It provides new insights into non-pharmacological tailored activities by developing a rating scheme for the level of personalisation and tested its validity by investigating the effectiveness of interventions with different levels of tailoring on BPSD and QoL. Healthcare professionals and practitioners can use our findings to tailor interventions to benefit patients' outcomes. We recommend the application of structural and comprehensive assessment 
approaches to identify and address two or more PWD characteristics (capacities, preferences, habits and living environment, etc) in designing tailored activities, and allow interventionists to use their professional judgement to modify the interventions to respond to spontaneous needs of PWD to develop tailored activities with a high level of personalisation.

Our systematic review has implications for future intervention research. Fourteen studies had no more than 20 participants for each arm, and only 10 RCTs were judged as low risk. Evaluation studies should adhere to current methodological standards, for example, a randomised and concealed allocation, adequate blinding (at least participants and outcome assessors), and recruitment of adequate samples. ${ }^{23}$

\section{CONCLUSION}

This systematic review shows that tailored activities slightly reduced BPSD and depression, had a small effect on improving QoL and had large effects on facilitating the level of engagement among PWD. Additionally, we advanced existing literature by proposing and testing the validity of a rating scheme for the level of personalisation. Additional high-quality tailored intervention studies with sufficient samples are needed.

\section{Author affiliations}

${ }^{1}$ Department of Social and Behavioural Sciences, City University of Hong Kong, Hong Kong, Hong Kong, Hong Kong

${ }^{2}$ Department of Social Work and Social Administration, University of Hong Kong, Hong Kong, Hong Kong

${ }^{3}$ Department of Social Work and Social Administration, Sau Po Centre on Ageing, University of Hong Kong, Hong Kong, Hong Kong

\section{Twitter Terry Lum @TerryLum}

Contributors SL was responsible for the overall content of the systematic review. SL wrote the systematic review, performed the preliminary searches and data extraction, conducted quality assessments and drafted the systematic review paper. SL, AYZ, T Liu and JCPC designed the rating scheme for the level of personalisation. MSLM cross-checked data extraction and performed quality ratings independently. GW and T Lum made substantial contributions to the conception and design of the systematic review and assisted SL, AYZ, T Liu and JCPC to resolve any discrepancies regarding study inclusion, data extraction and quality ratings. All authors offered critical revisions for the systematic review manuscript.

Funding This work was supported by a donation from Tin Hing-Sin Sam to promote non-pharmacological interventions for people with dementia and their caregivers in the community.

Competing interests None declared.

Patient consent for publication Not applicable.

Ethics approval This study does not involve human participants.

Provenance and peer review Not commissioned; externally peer reviewed.

Data availability statement Data are available upon reasonable request. All data relevant to the study are included in the article or uploaded as supplementary information.

Supplemental material This content has been supplied by the author(s). It has not been vetted by BMJ Publishing Group Limited (BMJ) and may not have been peer-reviewed. Any opinions or recommendations discussed are solely those of the author(s) and are not endorsed by BMJ. BMJ disclaims all liability and responsibility arising from any reliance placed on the content. Where the content includes any translated material, BMJ does not warrant the accuracy and reliability of the translations (including but not limited to local regulations, clinical guidelines, terminology, drug names and drug dosages), and is not responsible for any error and/or omissions arising from translation and adaptation or otherwise.

Open access This is an open access article distributed in accordance with the Creative Commons Attribution Non Commercial (CC BY-NC 4.0) license, which permits others to distribute, remix, adapt, build upon this work non-commercially, and license their derivative works on different terms, provided the original work is properly cited, appropriate credit is given, any changes made indicated, and the use is non-commercial. See: http://creativecommons.org/licenses/by-nc/4.0/.

ORCID iDs

Shiyu Lu http://orcid.org/0000-0002-9355-4883

Gloria Wong http://orcid.org/0000-0002-1331-942X

Terry Lum http://orcid.org/0000-0003-1196-5345

\section{REFERENCES}

1 World Health Organization. Dementia, 2019. Available: https://www. who.int/news-room/fact-sheets/detail/dementia

2 Kales HC, Gitlin LN, Lyketsos CG. Assessment and management of behavioral and psychological symptoms of dementia. BMJ 2015;350:h369.

3 Gitlin LN, Kales HC, Lyketsos CG. Nonpharmacologic management of behavioral symptoms in dementia. JAMA 2012;308:2020-9.

4 Oliveira AMde, Radanovic M, Mello PCHde, et al. Nonpharmacological interventions to reduce behavioral and psychological symptoms of dementia: a systematic review. Biomed Res Int 2015;2015:1-9.

5 NICE. Dementia: assessment, management and support for people living with dementia and their carers [NG97], 2018. Available: https:// www.nice.org.uk/guidance/ng97

6 NICE. Dementia: Quality standard [QS184], 2019. Available: https://www.scie-socialcareonline.org.uk/dementia-qs-184/r/ a110f00000THdtRAAT

7 Trahan MA, Kuo J, Carlson MC, et al. A systematic review of strategies to foster activity engagement in persons with dementia. Health Educ Behav 2014;41:70S-83.

8 Testad I, Corbett A, Aarsland D, et al. The value of personalized psychosocial interventions to address behavioral and psychological symptoms in people with dementia living in care home settings: a systematic review. Int Psychogeriatr 2014;26:1083-98.

9 Travers C, Brooks D, Hines S, et al. Effectiveness of meaningful occupation interventions for people living with dementia in residentia aged care: a systematic review. JBI Database System Rev Implement Rep 2016;14:163-225.

10 Möhler R, Renom A, Renom H, et al. Personally tailored activities for improving psychosocial outcomes for people with dementia in longterm care. Cochrane Database Syst Rev 2018:2:CD009812.

11 Möhler R, Renom A, Renom H, et al. Personally tailored activities for improving psychosocial outcomes for people with dementia in community settings. Cochrane Database Syst Rev 2020;8:CD010515.

12 Möhler R, Renom A, Renom H. Personally tailored activities for people with dementia living in their own homes. Cochrane Database Syst Rev 2020;8.

13 Bennett S, Laver K, Voigt-Radloff S, et al. Occupational therapy for people with dementia and their family carers provided at home: a systematic review and meta-analysis. BMJ Open 2019;9:e026308.

14 Macaulay S. The broken lens of BPSD: why we need to rethink the way we label the behavior of people who live with alzheimer disease. J Am Med Dir Assoc 2018;19:177-80.

15 Chenoweth L, King MT, Jeon Y-H, et al. Caring for aged dementia care resident study (CADRES) of person-centred care, dementia-care mapping, and usual care in dementia: a cluster-randomised trial. Lancet Neurol 2009;8:317-25.

16 Gitlin LN, Winter L, Burke J, et al. Tailored activities to manage neuropsychiatric behaviors in persons with dementia and reduce caregiver burden: a randomized pilot study. Am J Geriatr Psychiatry 2008;16:229-39.

17 Page MJ, McKenzie JE, Bossuyt PM, et al. The PRISMA 2020 statement: an updated guideline for reporting systematic reviews. BMJ 2021;372:n71.

18 Cummings JL, Mega M, Gray K, et al. The neuropsychiatric inventory: comprehensive assessment of psychopathology in dementia. Neurology 1994;44:2308-14.

19 Alexopoulos GS, Abrams RC, Young RC, et al. Cornell scale for depression in dementia. Biol Psychiatry 1988;23:271-84.

20 Cohen-Mansfield J, Billig N. Agitated behaviors in the elderly. I. a conceptual review. J Am Geriatr Soc 1986;34:711-21. 
21 Cochrane Effective Practice and Organisation of Care (EPOC). Describing interventions in EPOC reviews, 2017. Available: https:// epoc.cochrane.org/resources/epoc-resources-review-authors

22 Cohen J. Statistical power analysis for the behavioral sciences. Academic Press, 2013.

23 Higgins JP, Green S. Cochrane handbook for systematic reviews of interventions. Chichester: John Wiley \& Sons, 2011.

24 Higgins JP, Li T, Deeks JJ. Choosing effect measures and computing estimates of effect: the cochrane collaboration, 2019. Available: www.training.cochrane.org/handbook

25 Sterne JAC, Savović J, Page MJ, et al. Rob 2: a revised tool for assessing risk of bias in randomised trials. BMJ 2019;2:14898

26 Lam LCW, Lui VWC, Luk DNY, et al. Effectiveness of an individualized functional training program on affective disturbances and functional skills in mild and moderate dementia-a randomized control trial. Int $J$ Geriatr Psychiatry 2010;25:133-41.

27 Holthoff VA, Marschner K, Scharf M, et al. Effects of physical activity training in patients with alzheimer's dementia: results of a pilot RCT study. PLoS One 2015;10:e0121478.

28 Telenius EW, Engedal K, Bergland A. Effect of a high-intensity exercise program on physical function and mental health in nursing home residents with dementia: an assessor blinded randomized controlled trial. PLoS One 2015;10:e0126102.

29 Davison TE, Nayer K, Coxon S, et al. A personalized multimedia device to treat agitated behavior and improve mood in people with dementia: a pilot study. Geriatr Nurs 2016;37:25.

30 Giuli C, Papa R, Lattanzio F, et al. The effects of cognitive training for elderly: results from my mind project. Rejuvenation Res 2016;19:485-94.

31 Garland K, Beer E, Eppingstall B, et al. A comparison of two treatments of agitated behavior in nursing home residents with dementia: simulated family presence and preferred music. Am J Geriatr Psychiatry 2007;15:514-21.

32 Sung H-C, Chang AM, Lee W-L. A preferred music listening intervention to reduce anxiety in older adults with dementia in nursing homes. J Clin Nurs 2010;19:1056-64.

33 Lin Y, Chu H, Yang C-Y, et al. Effectiveness of group music intervention against agitated behavior in elderly persons with dementia. Int J Geriatr Psychiatry 2011;26:670-8.

34 Ridder HMO, Stige B, Qvale LG, et al. Individual music therapy for agitation in dementia: an exploratory randomized controlled trial. Aging Ment Health 2013;17:667-78.

35 Sakamoto M, Ando H, Tsutou A. Comparing the effects of different individualized music interventions for elderly individuals with severe dementia. Int Psychogeriatr 2013;25:775-84.

$36 \mathrm{Kwak} \mathrm{J}$, Anderson K. Findings from a prospective randomized controlled trial of an individualized music listening program for persons with dementia. J App/ Gerontol 2020;39:567-75.

37 Weise L, Töpfer NF, Deux J, et al. Feasibility and effects of individualized recorded music for people with dementia: a pilot RCT study. Nordic J Music Ther 2020;29:39-56.

38 Orsulic-Jeras S, Judge KS, Camp CJ. Montessori-based activities for long-term care residents with advanced dementia: effects on engagement and affect. Gerontologist 2000;40:107-11.

39 Cohen-Mansfield J, Parpura-Gill A, Golander H. Utilization of selfidentity roles for designing interventions for persons with dementia. $J$ Gerontol B Psychol Sci Soc Sci 2006;61:P202-12.

40 Cohen-Mansfield J, Libin A, Marx MS. Nonpharmacological treatment of agitation: a controlled trial of systematic individualized intervention. J Gerontol A Biol Sci Med Sci 2007;62:908-16.

41 Kolanowski A, Litaker M, Buettner L, et al. A randomized clinical trial of theory-based activities for the behavioral symptoms of dementia in nursing home residents. J Am Geriatr Soc 2011;59:1032-41.

42 van der Ploeg ES, Eppingstall B, Camp CJ, et al. A randomized crossover trial to study the effect of personalized, one-to-one interaction using Montessori-based activities on agitation, affect, and engagement in nursing home residents with dementia. Int Psychogeriatr 2013;25:565-75.

43 Yoon JE, Lee SM, Lim HS, et al. The effects of cognitive activity combined with active extremity exercise on balance, walking activity, memory level and quality of life of an older adult sample with dementia. J Phys Ther Sci 2013;25:1601-4.

44 Toba $\mathrm{K}$, Nakamura $\mathrm{Y}$, Endo $\mathrm{H}$, et al. Intensive rehabilitation for dementia improved cognitive function and reduced behavioral disturbance in geriatric health service facilities in Japan. Geriatr Gerontol Int 2014;14:206-11.

45 Lu YY-F, Bakas T, Yang Z, et al. Feasibility and effect sizes of the revised daily engagement of meaningful activities intervention for individuals with mild cognitive impairment and their caregivers. $J$ Gerontol Nurs 2016;42:45-58.
46 Prick A-E, Scherder E, et al. The effects of a multicomponent dyadic intervention on the mood, behavior, and physical health of people with dementia: a randomized controlled trial. Clin Interv Aging 2016;11:383-95.

47 Li D-M, Li X-X. The effect of folk recreation program in improving symptoms: a study of Chinese elder dementia patients. Int J Geriatr Psychiatry 2017;32:901-8.

48 Tanaka S, Honda S, Nakano H, et al. Comparison between group and personal rehabilitation for dementia in a geriatric health service facility: single-blinded randomized controlled study. Psychogeriatrics 2017; 17:177-85.

49 Jeon Y-H, Krein L, Simpson JM, et al. Feasibility and potential effects of interdisciplinary home-based reablement program (I-HARP) for people with cognitive and functional decline: a pilot trial. Aging Ment Health 2020;24:1916-1925.

50 Huber A, Oppikofer S, Meister L, et al. Music \& memory: the impact of individualized music listening on depression, agitation, and positive emotions in persons with dementia. Act Adapt Aging 2021;45:70-84.

51 Gitlin LN, Winter L, Dennis MP, et al. A biobehavioral home-based intervention and the well-being of patients with dementia and their caregivers: the cope randomized trial. JAMA 2010;304:983-91.

52 Bailey EM, Stevens AB, LaRocca MA, et al. A randomized controlled trial of a therapeutic intervention for nursing home residents with dementia and depressive symptoms. J Appl Gerontol 2017;36:895-908.

53 Gitlin LN, Arthur P, Piersol C, et al. Targeting behavioral symptoms and functional decline in dementia: a randomized clinical trial. J Am Geriatr Soc 2018;66:339-45.

54 Novelli MMPC, Machado SCB, Lima GB, et al. Effects of the tailored activity program in Brazil (TAP-BR) for persons with dementia: a randomized pilot trial. Alzheimer Dis Assoc Disord 2018;32:339-45.

55 de Oliveira AM, Radanovic M, Homem de Mello PC, et al. An intervention to reduce neuropsychiatric symptoms and caregiver burden in dementia: preliminary results from a randomized trial of the tailored activity program-outpatient version. Int J Geriatr Psychiatry 2019;34:1301-7.

56 O'Connor CM, Clemson L, Brodaty $\mathrm{H}$, et al. The tailored activity program (TAP) to address behavioral disturbances in frontotemporal dementia: a feasibility and pilot study. Disabil Rehabil 2019;41:299-310.

57 Van Haitsma KS, Curyto K, Abbott KM, et al. A randomized controlled trial for an individualized positive psychosocial intervention for the affective and behavioral symptoms of dementia in nursing home residents. J Gerontol B Psychol Sci Soc Sci 2015;70:35-45.

58 Dechamps A, Alban R, Jen J, et al. Individualized cognition-action intervention to prevent behavioral disturbances and functional decline in institutionalized older adults: a randomized pilot trial. Int $J$ Geriatr Psychiatry 2009;25:850-60.

59 Cohen-Mansfield J, Thein K, Marx MS, et al. Efficacy of nonpharmacologic interventions for agitation in advanced dementia: a randomized, placebo-controlled trial. J Clin Psychiatry 2012;73:1255-61.

60 Hall GR, Buckwalter KC. Progressively lowered stress threshold: a conceptual model for care of adults with Alzheimer's disease. Arch Psychiatr Nurs 1987;1:399-406.

61 Cohen-Mansfield J, Werner P, Marx MS. An observational study of agitation in agitated nursing home residents. Int Psychogeriatr 1989;1:153-65.

62 Logsdon RG, Teri L, Weiner MF, et al. Assessment of agitation in alzheimer's disease: the agitated behavior in dementia scale. J Am Geriatr Soc 1999;47:1354-8.

63 Reisberg B, Borenstein J, Salob SP, et al. Behavioral symptoms in alzheimer's disease: phenomenology and treatment. J Clin Psychiatry 1987;48 Suppl:9-15.

64 Machida A. [Estimation of the reliability and validity of the short version of the 28 -item dementia behavior disturbance scale]. Nihon Ronen Igakkai Zasshi 2012;49:463-7.

65 Logsdon RG, Gibbons LE, McCurry SM. Quality of life in Alzheimer's disease: patient and caregiver reports. J Ment Health Aging 1999;5:21-32 https://www.researchgate.net/publication/232417911 Quality_of_Life_in_Alzheimer's_disease_Patient_and_Caregiver_ Reports

66 EuroQol Group. EuroQol--a new facility for the measurement of health-related quality of life. Health Policy 1990;16:199-208.

67 Terada S, Ishizu H, Fujisawa Y, et al. Development and evaluation of a health-related quality of life questionnaire for the elderly with dementia in Japan. Int J Geriatr Psychiatry 2002;17:851-8.

68 Yesavage JA. Geriatric depression scale. Psychopharmacol Bull 1988:24:709-11. 
69 Helmes E, Csapo KG, Short JA. Standardization and validation of the multidimensional observation scale for elderly subjects (moses). J Gerontol 1987;42:395-405.

70 Kroenke K, Spitzer RL, Williams JB. The PHQ-9: validity of a brief depression severity measure. J Gen Intern Med 2001;16:606-13.
71 Skrajner MJ, Camp CJ. Resident-assisted

Montessoriprogramming (RAMP ${ }^{\mathrm{TM}}$ ): use of a small group reading activity run by persons with dementia in adult day health care and long-term care settings. Am J Alzheimers Dis Other Demen $2007 ; 22: 27-36$ 